\title{
Nonovershooting linear multivariable state feedback tracking controllers
}

\author{
Robert Schmid ${ }^{* *}$ Lorenzo Ntogramatzidis ${ }^{* * *}$ \\ Department of Electrical and Electronic Engineering, The University \\ of Melbourne, Parkville, Vic. 3010, Australia \\ **(e-mail: schmid@ee.unimelb.edu.au). \\ *** (e-mail:lnt@ee.unimelb.edu.au)
}

\begin{abstract}
We consider the use of linear multivariable feedback control to achieve a nonovershooting step response. A method is given for designing an LTI state feedback controller to asymptotically track a constant step reference with no overshoot and arbitrarily small rise time. Results are given for both minimum phase and nonminimum phase multivariable LTI systems.
\end{abstract}

\section{INTRODUCTION}

The problem of obtaining a linear feedback controller for a linear time invariant (LTI) plant to ensure the tracking response to a step input converges to a desired step reference without overshoot has received some attention recently. For continuous time systems, Darbha and Bhattacharyya (2003) gave a two parameter linear feedback controller to render the step response nonovershooting. Bement and Jayasuriya (2004) gave an eigenstructure assignment method to obtain a nonovershooting linear state feedback controller for plants with one nonminimum phase (right hand complex plane) zero. Darbha (2003) gave conditions for the existence of a linear controller to achieve a sign invariant impulse response, and hence also a nonovershooting step response. Corresponding conditions for discrete systems were given in Darbha and Bhattacharyya (2002). Recently Krstic and Bement (2006) considered strict feedback nonlinear systems and used a backstepping approach to convert the system to an nonovershooting linear system.

A common feature of all these papers is that they considered single input single output (SISO) systems, and that the system state was assumed to be initially at rest. In this paper we consider multiple input multiple output (MIMO) systems, and use linear state feedback control to design a nonovershooting controller for a step reference. We will show that for a minimum phase MIMO system, a linear state feedback controller can be obtained to asymptotically track a step reference with no overshoot, from all initial conditions. The controller can be readily chosen to achieve any desired convergence rate (settling time). For nonminimum phase MIMO systems we design a state feedback controller and give a set of initial conditions from which the tracking controller will achieve guaranteed no overshoot. The design methods make use of the combined eigenvalue and eigenvector placement methods given in Moore (1976).

\section{PROBLEM FORMULATION}

We consider the multi-input multi-output (MIMO) continuous time system $\Sigma$ characterized by

$$
\Sigma:\left\{\begin{array}{l}
\dot{x}(t)=A x(t)+B u(t), x(0)=x_{0} \in \mathbb{R}^{n} \\
y(t)=C x(t),
\end{array}\right.
$$

where, for all $t \geq 0, x(t) \in \mathbb{R}^{n}, u(t) \in \mathbb{R}^{m}$, and $y(t) \in \mathbb{R}^{p}$, are respectively the state, the control input, and the output of the system; $A, B$, and $C$ are appropriate dimensional constant matrices with $\operatorname{rank}(B)=m, \operatorname{rank}(C)=p$, and $p \leq m$. In this paper we make the following standing assumptions:

Assumption 2.1. The system $\Sigma$ is right-invertible, stabilizable and has no invariant zeros at the origin.

The following method for designing a tracking controller for a constant step reference $r \in \mathbb{R}^{p}$ is standard: choose a feedback gain matrix $F$ such that $A-B F$ is a stability matrix (all eigenvalues lie in the left hand complex plane (LHP)). By virtue of Assumption 2.1, two vectors $x_{s s}$ and $u_{s s}$ always exist satisfying

$$
\begin{aligned}
& 0=A x_{s s}+B u_{s s} \\
& r=C x_{s s}
\end{aligned}
$$

for any $r \in \mathbb{R}^{p}$. If $\Sigma$ is also left invertible, the solutions $x_{s s}$ and $u_{s s}$ satisfying (2)-(3) are uniquely given by

$$
\left[\begin{array}{l}
x_{s s} \\
u_{s s}
\end{array}\right]=\left[\begin{array}{ll}
A & B \\
C & 0
\end{array}\right]^{-1}\left[\begin{array}{l}
0 \\
r
\end{array}\right] .
$$

Applying the state feedback control law

$$
u=F\left(x_{s s}-x\right)+u_{s s}
$$

to (1) yields the closed loop system

$$
\Sigma_{c l}:\left\{\begin{array}{l}
\dot{x}(t)=(A-B F) x(t)+B\left(u_{s s}+F x_{s s}\right), x(0)=x_{0}(6) \\
y(t)=C x(t),
\end{array}\right.
$$

which is equivalent, up to the changes of variable $\xi:=x_{\mathrm{ss}}-$ $x$, to the homogeneous system

$$
\Sigma_{\text {hom }}: \quad\left\{\begin{array}{l}
\dot{\xi}(t)=(A-B F) \xi(t), \quad \xi(0)=x_{\mathrm{ss}}-x_{0} \\
y(t)=-C \xi(t)+r .
\end{array}\right.
$$

Since $A-B F$ is stable, it follows that $x$ converges to $x_{\mathrm{ss}}$ and $y(t)$ converges to $r$ asymptotically as $t$ goes to infinity.

The tracking problem we address in this paper is how to choose the gain matrix $F$ such that the output $y$ tracks 
the step reference $r$ without overshoot. We formally define the nonovershooting property as follows:

Definition 2.1. Let $r \in \mathbb{R}^{p}$ be a constant tracking reference. The system $\Sigma$ has a nonovershooting response for $r$ from the initial condition $x_{0} \in \mathbb{R}^{n}$ if the output $y$ of (1) arising from $x_{0}$ yields a tracking error $\varepsilon(t)=r-y(t)$ that satisfies

(1) $\varepsilon(t) \rightarrow 0$ as $t \rightarrow \infty$, and

(2) $\varepsilon(t)$ has no changes of sign in any component, i.e., for each $i \in\{1, \ldots, p\}, \operatorname{sgn}\left(\varepsilon_{i}(t)\right)$ is constant for all $t \geq 0$.

\section{DESIGN OF NON-OVERSHOOTING FEEDBACK CONTROLLERS}

Firstly we consider how to obtain a nonovershooting control law for systems subject to the following assumption (in addition to Assumption 2.1):

Assumption 3.1. $\Sigma$ is a left invertible system.

Thus $\Sigma$ is invertible and square $(m=p)$. A key result in the design of our nonovershootng controller will be the results on simultaneous eigenvalue and eigenvector assignment given in the classical paper Moore (1976), which we summarize as follows.

Lemma 1. Let $\mathcal{L}=\left\{\lambda_{1}, \ldots, \lambda_{n}\right\}$ be a self conjugate set of $n$ distinct complex numbers. Let $\mathcal{S}=\left\{s_{1}, \ldots, s_{n}\right\} \subset \mathbb{R}^{p}$ be a set of $n$ (not necessarily distinct) vectors in $\mathbb{R}^{p}$. Assume for $1 \leq i \leq n$, the matrix equation

$$
\left[\begin{array}{cc}
\lambda_{i} I-A-B \\
C & 0
\end{array}\right]\left[\begin{array}{c}
v_{i} \\
w_{i}
\end{array}\right]=\left[\begin{array}{c}
0 \\
s_{i}
\end{array}\right]
$$

has solutions sets $\mathcal{V}=\left\{v_{1}, \ldots, v_{n}\right\} \subset \mathbb{C}^{n}$ and $\mathcal{W}=$ $\left\{w_{1}, \ldots, w_{n}\right\} \subset \mathbb{C}^{p}$. Then provided the set $\mathcal{V}$ is a linearly independent subset of $\mathbb{C}^{n}$, there exists a unique real feedback matrix $F$ such that, for all $i \in\{1, \ldots, n\}$,

$$
(A-B F) v_{i}=\lambda_{i} v_{i}
$$

Proof: The sets $\mathcal{L}$ and $\mathcal{V}$ satisfy the assumptions of Proposition 1 of Moore (1976), and hence the results of that paper may be used to construct a feedback matrix $F$ to satisfy (9).

Our first method for designing nonovershooting controllers will apply to systems that satisfy (in addition to Assumptions 2.1 and 3.1 )

Assumption 3.2. $\Sigma$ has $n-p$ distinct LHP zeros.

A necessary and sufficient condition for Assumption 3.2 is that $\Sigma$ is of minimum phase and $\operatorname{det}(C B) \neq 0$, Maciejowski (1989). The design method for our nonovershooting control law is as follows. Let $\mathcal{L}=\left\{\lambda_{1}, \ldots, \lambda_{n}\right\} \subset \mathbb{C}$ be the distinct LHP eigenvalues of $A-B F$ to be chosen. Let $\left\{z_{i}: i \in\{1, \ldots, n-p\}\right\} \subset \mathbb{C}$ be the $n-p$ distinct zeros of $\Sigma$. Then we choose $\lambda_{i}=z_{i}$ for $i \in\{1, \ldots, n-p\}$; these modes are stable as the $z_{i}$ all lie in the LHP by assumption. By Lemma 7 , if $\Sigma$ has any uncontrollable stable modes these will also be invariant zeros and will be included among the $\lambda_{i}$ for $i \in\{1, \ldots, n-p\}$. For $i \in\{n-p+1, \ldots, n\}$, the $\lambda_{i}<0$ may be freely chosen to be any real distinct stable modes.

Next let $\left\{e_{1}, \ldots, e_{p}\right\}$ be the standard basis vectors for $\mathbb{R}^{p}$, and let $\mathcal{S}=\left\{s_{1}, \ldots, s_{n}\right\} \subset \mathbb{R}^{p}$ be such that

$$
s_{i}=\left\{\begin{array}{cl}
0 & \text { for } i \in\{1, \ldots, n-p\} \\
e_{1} & \text { for } i=n-p+1 \\
\vdots & \\
e_{p} & \text { for } i=n .
\end{array}\right.
$$

Let $P_{\Sigma}(s):=\left[\begin{array}{cc}s I-A & -B \\ C & 0\end{array}\right]$ be the system matrix pencil. Since $\Sigma$ is invertible, the rank of $P_{\Sigma}(s)$ is $n+p$ if and only if $s \in \mathbb{C}$ is not an invariant zero of $\Sigma$. Since $\left\{\lambda_{i}: i \in\right.$ $\{1, \ldots, n-p\}\}$ are the distinct zeros of $\Sigma$, the kernels of $P_{\Sigma}\left(\lambda_{i}\right)$ for all $i \in\{1, \ldots, n-p\}$ are 1-dimensional subspaces of $\mathbb{C}^{n+p}$, while ker $P_{\Sigma}\left(\lambda_{i}\right)=\{0\}$ for all $i \in\{n-$ $p+1, \ldots, n\}$. Solving (8) for the vectors in $\mathcal{S}$, we obtain $\mathcal{V}=\left\{v_{1}, \ldots, v_{n}\right\} \subset \mathbb{C}^{n}$ and $\mathcal{W}=\left\{w_{1}, \ldots, w_{n}\right\} \subset \mathbb{C}^{p}$ given by

$\left[\begin{array}{c}v_{i} \\ w_{i}\end{array}\right]= \begin{cases}\operatorname{ker} P_{\Sigma}\left(\lambda_{i}\right) & \text { for } i \in\{1, \ldots, n-p\}, \\ P_{\Sigma}^{-1}\left(\lambda_{i}\right)\left[\begin{array}{c}0 \\ e_{i-(n-p)}\end{array}\right] & \text { for } i \in\{n-p+1, \ldots, n\} .\end{cases}$

Thus the vectors in $\mathcal{V}$ satisfy $s_{i}=C v_{i}$ for all $i \in\{1, \ldots, n\}$. Then provided $\mathcal{V}$ is linearly independent, by Lemma 1 , the procedure in Moore (1976) can then be applied to obtain $F$ such that $A-B F$ has distinct eigenvalues and corresponding linearly independent eigenvectors given by $\mathcal{L}$ and $\mathcal{V}$ respectively. Now Theorem 2 says that the feedback gain matrix $F$ can be used to obtain a state feedback control law that will yield a closed loop system response that will converge to any given step reference $r$ without overshoot, from all initial conditions.

Theorem 2. Assume that $\Sigma$ satisfies Assumption 2.1, 3.1 and 3.2, and $F$ is defined as above. Let $r \in \mathbb{R}^{p}$ be any step reference, and apply $u$ as in (5) to $\Sigma$. Then the output $y(t)$ from $\Sigma_{c l}$ will be non overshooting for all initial conditions $x_{0} \in \mathbb{R}^{n}$.

Proof: Applying $u$ as in (5) to $\Sigma$ and introducing state coordinates $\xi:=x_{\mathrm{ss}}-x$ yields the homogeneous system $\Sigma_{\text {hom }}$ in (7). For any initial condition $\xi_{0} \in \mathbb{R}^{n}$, the tracking error $\varepsilon(t)=C \xi(t)$ is given by

$$
\varepsilon(t)=C e^{(A-B F) t} \xi_{0} .
$$

As the eigenvectors in $\mathcal{V}$ are linearly independent, the matrix $V:=\left[\begin{array}{llll}v_{1} & v_{2} & \ldots & v_{n}\end{array}\right]$ is invertible. Introduce $\alpha:=\left[\begin{array}{llll}\alpha_{1} & \alpha_{2} & \ldots & \alpha_{n}\end{array}\right]^{\top^{2}}=V^{-1} \xi_{0}$. It follows that the tracking error can be expressed by

$$
\varepsilon(t)=\sum_{i=1}^{n} C v_{i} \alpha_{i} e^{\lambda_{i} t}=\sum_{i=n-p+1}^{n} e_{i-(n-p)} \alpha_{i} e^{\lambda_{i} t} .
$$

As the eigenvalues all lie in the LHP, the system $\Sigma_{h o m}$ is asymptotically stable, and $\varepsilon(t)=C \xi(t) \rightarrow 0$. As the $e_{i}$ vectors are orthogonal, each component of $\varepsilon(t)$ contains exactly one mode. Hence $\varepsilon(t)$ cannot change sign in any component, and $y(t)$ converges to $r$ without overshoot. $\square$

Remark 3.1. For any given choice of $\mathcal{L}$ and $\mathcal{S}$, it is not assured that the vectors in $\mathcal{V}$ obtained from solving (8) will be linearly independent. If the vectors in $\left\{v_{1}, \ldots, v_{n-p}\right\}$ are linearly dependent, then it will not be possible to obtain a suitable $F$. However if the vectors in $\mathcal{V}$ up to $\left\{v_{1}, \ldots, v_{n-p}\right\}$ are independent, but independence of $\mathcal{V}$ fails due to the vectors $v_{i}$ for $1 \in\{n-p+1, \ldots, n\}$, there are then $p$ ! possible ways to re-order the $p$ standard basis vectors in $\mathcal{S}$ and resolve (8) to obtain a new $\mathcal{V}$. If this procedure failed to obtain a linearly independent $\mathcal{V}$, we could alter one of 
the eigenvalues in $\mathcal{L}$, for $1 \in\{n-p+1, n\}$, and again solve (8).

Remark 3.2. As the $\lambda_{i}$ for $1 \in\{n-p+1, \ldots, n\}$ can be freely chosen to be any distinct LHP real numbers (provided they are distinct from the stable zeros of $\Sigma$, and provided also that the resulting $\mathcal{V}$ is linearly independent), the rate of convergence of the output trajectory to the target reference can be chosen to be arbitrarily fast. Note also that $F$ is independent of both $r$ and $x_{0}$. Hence the same $F$ can be used to achieve nonovershooting convergence for any $r \in \mathbb{R}^{p}$ and any $x_{0} \in \mathbb{R}^{n}$. The values of $r$ and $x_{0}$ enter the control law $u$ only through the values of $x_{\mathrm{ss}}$ and $u_{\mathrm{ss}}$.

The main limitation of Theorem 2 is that it requires $\Sigma$ to have $n-p$ LHP zeros, and many systems of interest will not satisfy this requirement. Our next theorem shows that we can replace Assumption 3.2 with

Assumption 3.3. The system $\Sigma$ has at least $n-2 p$ distinct LHP zeros.

Note that a system satisfying Assumption 3.3 may be nonminimum phase with up to $p$ zeros in the RHP. The design method for $F$ is as follows.

Let $\mathcal{L}=\left\{\lambda_{1}, \ldots, \lambda_{n}\right\}$ be the distinct stable eigenvalues of $A-B F$ to be chosen. Let $z_{1}, \ldots, z_{n-2 p}$ be the $n-$ $2 p$ distinct invariant LHP zeros of $\Sigma$. Mimicking the procedure for Theorem 2, we choose $\lambda_{i}=z_{i}$ for $i \in$ $\{1, \ldots, n-2 p\}$, and for $i \in\{n-2 p+1, \ldots, n\}$ the $\lambda_{i}$ may be freely chosen to be any distinct real stable modes.

Next let $\mathcal{S}=\left\{s_{1}, \ldots, s_{n}\right\} \subset \mathbb{R}^{p}$ be such that

$$
s_{i}=\left\{\begin{array}{cl}
0 & \text { for } i \in\{1, \ldots, n-2 p\} \\
e_{1} & \text { for } i \in\{n-2 p+1, n-2 p+2\} \\
e_{2} & \text { for } i \in\{n-2 p+3, n-2 p+4\} \\
\vdots & \\
e_{p} & \text { for } i \in\{n-1, n\} .
\end{array}\right.
$$

where $\left\{e_{1}, \ldots, e_{p}\right\}$ is the standard basis for $\mathbb{R}^{p}$. Solving (8) for the vectors in $\mathcal{S}$, we obtain $\mathcal{V}=\left\{v_{1}, \ldots, v_{n}\right\} \subset \mathbb{C}^{n}$ and $\mathcal{W}=\left\{w_{1}, \ldots, w_{n}\right\} \subset \mathbb{C}^{p}$ given by

$$
\left[\begin{array}{c}
v_{i} \\
w_{i}
\end{array}\right]=\left\{\begin{array}{l}
\operatorname{ker} P_{\Sigma}\left(\lambda_{i}\right) \quad \text { for all } i \in\{1, \ldots, n-2 p\} \\
P_{\Sigma}^{-1}\left(\lambda_{i}\right)\left[\begin{array}{c}
0 \\
s_{i}
\end{array}\right] \text { for all } i \in\{n-2 p+1, \ldots, n\} .
\end{array}\right.
$$

Then provided $\mathcal{V}$ is linearly independent, by Lemma 1 , the procedure in Moore (1976) can then be applied to obtain $F$ such that $A-B F$ has distinct eigenvalues and corresponding linearly independent eigenvectors given by $\mathcal{L}$ and $\mathcal{V}$ respectively. The vectors in $\mathcal{V}$ satisfy $s_{i}=C v_{i}$ for all $i \in\{1, \ldots, n\}$. To simplify the notation, in the following we will use, for each $k \in\{1, \ldots, p\}, v_{k, 1}$ and $v_{k, 2}$ to denote be the eigenvectors in $\mathcal{V}$ associated with standard basis vector $e_{k}$ in (13). Thus for example $v_{1,1}=$ $v_{n-2 p+1}$ and $v_{1,2}=v_{n-2 p+2}$ because they are associated with $e_{1}=s_{n-2 p+1}=s_{n-2 p+2}$; likewise $v_{p, 1}=v_{n-1}$ and $v_{p, 2}=v_{n}$ because they are associated with $e_{p}=$ $s_{n-1}=s_{n}$. We will also use $\lambda_{k, 1}$ and $\lambda_{k, 2}$ to denote the corresponding eigenvalues. We assume without loss of generality that for each $k \in\{1, \ldots, p\}$, the eigenvalues are ordered increasingly, e.g. $\lambda_{k, 1}<\lambda_{k, 2}$.
Theorem 3 then gives a set of initial conditions from which, for given $r \in \mathbb{R}^{p}$, the closed loop system $\Sigma_{c l}$ will yield a nonovershooting step response.

Theorem 3. Assume that the system $\Sigma$ satisfies Assumption 3.3, and that $F$ has been designed as above. For each $k \in\{1, \ldots, p\}$, let $\mathcal{H}_{k}=\operatorname{span}\left\{v_{k, 1}, v_{k, 2}\right\}$ and let $\mathcal{J}_{k} \subseteq \mathcal{H}_{k}$ be the region

$$
\mathcal{J}_{k}:=\left\{\gamma_{k, 1} v_{k, 1}+\gamma_{k, 2}\left(v_{k, 1}-v_{k, 2}\right): \gamma_{k, 1} \cdot \gamma_{k, 2} \leq 0\right\}
$$

For any $x \in \mathbb{R}^{n}$, let $x_{k}$ denote the orthogonal projection of $x$ onto $\mathcal{H}_{k}$. Let $\mathcal{J} \subseteq \mathbb{R}^{n}$ consist of those points in $x \in \mathbb{R}^{n}$ for which $x_{k} \in \mathcal{J}_{k}$ for all $k \in\{1, \ldots, p\}$.

Let $r \in \mathbb{R}^{p}$ be given and let $x_{0} \in \mathbb{R}^{n}$ be an initial condition. The closed loop system $\Sigma_{c l}$ obtained from applying $u$ in (5) to $\Sigma$ will be nonovershooting for $x_{0}$ if and only if $x_{\mathrm{ss}}-x_{0} \in \mathcal{J}$.

Proof Introduce new state coordinates $\xi=x_{\mathrm{ss}}-x$ and assume $\xi_{0}=\xi(0) \in \mathcal{J}$. Introduce $\alpha:=\left[\begin{array}{llll}\alpha_{1} & \alpha_{2} & \ldots & \alpha_{n}\end{array}\right]^{\top}=$ $V^{-1} \xi_{0}$. From the proof of Theorem 2, it follows that the tracking error can be expressed by

$$
\varepsilon(t)=\sum_{i=n-2 p+1}^{n} e_{i-(n-p)} \alpha_{i} e^{\lambda_{i} t}
$$

As the eigenvalues all lie in the LHP, the system $\Sigma_{h o m}$ is asymptotically stable, and $\varepsilon(t)=C \xi(t) \rightarrow 0$. To see that $y(t)$ does not overshoot, note that $\xi_{0}$ may be expressed as

$$
\xi_{0}=\sum_{i=1}^{n-2 p} \alpha_{i} v_{i}+\sum_{k=1}^{p}\left(\alpha_{k, 1} v_{k, 1}+\alpha_{k, 2} v_{k, 2}\right),
$$

and hence $\xi_{0_{k}}$, the projection of $\xi_{0}$ onto $\mathcal{H}_{k}$, is

From (15)

$$
\xi_{0_{k}}=\alpha_{k, 1} v_{k, 1}+\alpha_{k, 2} v_{k, 2} \text {. }
$$

$$
\varepsilon(t)=\sum_{k=1}^{p} e_{k}\left(\alpha_{k, 1} e^{\lambda_{k, 1} t}+\alpha_{k, 2} e^{\lambda_{k, 2} t}\right) .
$$

Since the $e_{k}$ are orthogonal, we see that $\varepsilon(t)$ will not change sign if and only if, for every $k \in\{1, \ldots, p\}$,

$$
\varepsilon_{k}(t):=\alpha_{k, 1} e^{\lambda_{k, 1} t}+\alpha_{k, 2} e^{\lambda_{k, 2} t}
$$

does not change sign for $t \geq 0$. By assumption, since each $\xi_{0_{k}} \in \mathcal{J}_{k}$, we have $\gamma_{k, 1}$ and $\gamma_{k, 2}$ such that

$$
\xi_{0_{k}}=\gamma_{k, 1} v_{k, 1}+\gamma_{k, 2}\left(v_{k, 1}-v_{k, 2}\right)
$$

and $\gamma_{k, 1} \cdot \gamma_{k, 2} \leq 0$. Comparing (17) and (20), we have

$$
\left(\alpha_{k, 1}, \alpha_{k, 2}\right)=\left(\gamma_{k, 1}+\gamma_{k, 2},-\gamma_{k, 2}\right) .
$$

By assumption $\lambda_{k, 1}<\lambda_{k, 2}<0$. So by Lemma 6 , each $\varepsilon_{k}(t)$ does not change sign for $t \geq 0$. Thus $\varepsilon(t) \rightarrow 0$ without changing sign, and hence $y \rightarrow r$ without overshoot. $\square$.

Remark 3.3. In applying Theorem 3 to see if $\Sigma_{c l}$ is nonovershooting for a given $x_{0} \in \mathbb{R}^{n}$, we may construct the matrix

$$
P=\left[v_{1}|\ldots| v_{n-2 p}\left|v_{1,1}\right| v_{1,1}-v_{1,2}|\ldots| v_{p, 1} \mid v_{p, 1}-v_{p, 2}\right] \text {, }
$$

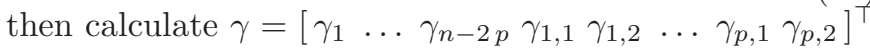
where

$$
\gamma=P^{-1}\left(x_{\mathrm{ss}}-x_{0}\right)
$$

and check if $\gamma_{k, 1} \gamma_{k, 2} \leq 0$ for all $k \in\{1, \ldots, p\}$. In particular, to see if the step response from the initial condition $x_{0}=0$ is nonovershooting for a given step reference $r$, we check whether $x_{\mathrm{ss}} \in \mathcal{J}$. 


\section{RELAXING THE LEFT-INVERTIBILITY ASSUMPTION}

Next we see how Assumption 3.1 can be relaxed, so that our design method may be applied to systems that are not left invertible.

To deal with non left-invertible systems, we recall Lemma 4 from Ntogramatzidis and Prattichizzo (2007). This Lemma gives feedback matrices $\Phi$ and $\Omega$ that can transform a non left-invertible system $\Sigma$ into a left-invertible square system $\hat{\Sigma}$, whose invariant zeros include those of the original system $\Sigma$, plus a set of values that can be chosen to lie in the LHP.

Before presenting Lemma 4, we need to recall some preliminary definitions and results (for details we refer to Basile and Marro (1992)). Let $\mathcal{V}^{\star}$ be the largest $(A, B)$ controlled invariant subspace contained in ker $C$. Recall that the feedback matrices $\Phi$ such that $(A+B \Phi) \mathcal{V}^{\star} \subseteq \mathcal{V}^{\star}$ are called friends of $\mathcal{V}^{\star}$. Dually, $\mathcal{S}^{\star}$ is the smallest $(A, C)$ conditioned invariant subspace containing $\operatorname{im} B$. The subspace $\mathcal{R}^{\star}=\mathcal{V}^{\star} \cap \mathcal{S}^{\star}$ denotes the reachability subspace on $\mathcal{V}^{\star}$. The eigenvalues of $(A+B \Phi)$ restricted to $\mathcal{V}^{\star}$, i.e. in $\sigma\left(A+B \Phi \mid \mathcal{V}^{\star}\right)$, are split into two sets: the eigenvalues of $\left(A+B \Phi \mid \mathcal{R}^{\star}\right)$ are assignable by a suitable choice of the friend $\Phi$. The eigenvalues in $\Gamma^{i n t} \triangleq \sigma\left(A+B \Phi \mid \mathcal{V}^{\star} / \mathcal{R}^{\star}\right)$ are fixed for all the choices of the friend $\Phi$; if $\Gamma^{i n t} \subset \mathbb{C}_{-}$, the subspace $\mathcal{V}^{\star}$ is said to be internally stabilizable. Similarly, by denoting with $\mathcal{R}_{0}$ the reachable subspace from the origin, the eigenvalues $\sigma\left(A+B \Phi \mid \mathbb{R}^{n} / \mathcal{V}^{\star}\right)$ are split into two sets: the eigenvalues of $\left(A+B \Phi \mid \mathcal{V}^{\star}+\mathcal{R}_{0} / \mathcal{V}^{\star}\right)$ are assignable by a suitable choice of the friend $\Phi$, whereas the eigenvalues in $\Gamma^{e x t} \triangleq \sigma\left(A+B \Phi \mid \mathbb{R}^{n} / \mathcal{V}^{\star}+\mathcal{R}_{0}\right)$ are fixed. If $\Gamma^{e x t} \subset \mathbb{C}_{-}, \mathcal{V}^{\star}$ is said to be externally stabilizable. The elements of $\Gamma^{i n t}$ are the invariant zeros of $\Sigma$.

Lemma 4. (Squaring Down Algorithm) Ntogramatzidis and Prattichizzo (2007)

Let $\Phi$ be a friend of $\mathcal{V}^{\star}$, i.e., $(A+B \Phi) \mathcal{V}^{\star} \subseteq \mathcal{V}^{\star}$, which assigns all the internal assignable poles of $\mathcal{V}^{\star}$ in the LHP, i.e., $\sigma\left(A+B \Phi \mid \mathcal{R}^{\star}\right) \subset \mathbb{C}^{-}$, where $\mathcal{R}^{\star}$ is the reachable subspace on $\mathcal{V}^{\star}$. Let $\Omega$ be a basis matrix of the subspace $\left(B^{-1} \mathcal{V}^{\star}\right)^{\perp}$ of the input space $\mathbb{R}^{m}$. The system $\hat{\Sigma}$ described by the triple $(A+B \Phi, B \Omega, C)$ is such that

- $\hat{\Sigma}$ is a left and right invertible square system with $p$ inputs and outputs;

- The invariant zeros of $\hat{\Sigma}$ are the union of those of $\Sigma$ and the internal poles of $\mathcal{V}^{\star}$ assigned through $\Phi$; they may be chosen to lie in the LHP.

Denote $\hat{A}=A+B \Phi$ and $\hat{B}=B \Omega$; then $\hat{\Sigma}$ can be represented by the triple $(\hat{A}, \hat{B}, C)$. If $\Sigma$ is such that $\operatorname{dim}\left(\mathcal{V}^{\star}\right)=n-p$, then $\hat{\Sigma}$ will be left and right-invertible, stabilizable, with no invariant zeros at the origin and $n-p$ LHP zeroes. Hence $\hat{\Sigma}$ satisfies Assumptions 2.1, 3.1 and 3.2 , and we can design the feedback gain matrix $\hat{F}$ for $\hat{\Sigma}$ by Theorem 2 and generalize as follows.

Theorem 5. Assume $\Sigma$ is such that the dimension of $\mathcal{V}^{\star}$ is $n-p$. Let $\hat{\Sigma}, \Omega, \Phi$ and $\hat{F}$ be as above. Computing

$$
\left[\begin{array}{l}
\hat{x}_{s s} \\
\hat{u}_{s s}
\end{array}\right]=\left[\begin{array}{ll}
\hat{A} & \hat{B} \\
C & 0
\end{array}\right]^{-1}\left[\begin{array}{l}
0 \\
r
\end{array}\right]
$$

and applying the control law

$$
u=\Omega \hat{F}\left(\hat{x}_{s s}-x\right)+\Phi x+\Omega \hat{u}_{s s}
$$

to $\Sigma$ yields $x \rightarrow \hat{x}_{s s}$ and $y \rightarrow r$ without overshoot.

Proof: Applying (25) to (1) yields the closed loop system

$$
\hat{\Sigma}_{c l}:\left\{\begin{array}{l}
\dot{x}(t)=(\hat{A}-\hat{B} \hat{F}) x(t)+\hat{B}\left(\hat{u}_{s s}+\hat{F} \hat{x}_{s s}\right), x(0)=x_{(26)} \\
y(t)=C x(t),
\end{array}\right.
$$

As $\hat{F}$ was defined with respect to $\hat{\Sigma}$, by Theorem 2 , we have $x \rightarrow \hat{x}_{s s}$ and $y \rightarrow r$ without overshoot.

Remark 4.1. A necessary and sufficient condition for $\operatorname{dim}\left(\mathcal{V}^{\star}\right)=n-p$ is $A$ ker $C \subseteq \operatorname{ker} C+\operatorname{im} B$.

Remark 4.2. If $\Sigma$ satisfies Assumption 2.1 and is such that $\operatorname{dim}\left(\mathcal{V}^{\star}\right) \geq n-2 p$, then $\hat{\Sigma}$ will satisfy Assumptions 2.1, 3.1 and 3.3. Hence we can design the feedback gain matrix $\hat{F}$ for $\hat{\Sigma}$ as in Theorem 3 and generalize Theorem 3 to the case where $\operatorname{dim}\left(\mathcal{V}^{\star}\right) \geq n-2 p$.

\section{EXAMPLES}

Example 1: Consider the MIMO system $\Sigma_{1}$ with

$A=\left[\begin{array}{cccc}1 & 0 & -2 & 4 \\ 3 & -3 & 0 & 1 \\ 1 & 2 & 0 & 1 \\ -1 & 2 & -4 & 5\end{array}\right], \quad B=\left[\begin{array}{ll}0 & 0 \\ 0 & 0 \\ 1 & 0 \\ 0 & 1\end{array}\right], \quad C=\left[\begin{array}{cccc}0 & 2 & 1 & 3 \\ 1 & -2 & 3 & 2\end{array}\right]$

Assume that the tracking target is $r=[3,-3]^{T}$. We see that the system has two invariant zeroes at $-0.857 \pm 3.543 i$ and open loop poles at $3.260 \pm 2.305 i,-2.803,-0.716$. Hence the system is minimum phase and square with $m=p=2$ and is open loop unstable.

Following the above design procedure, we choose closed loop poles of $\lambda_{1}=-0.857+3.543 i, \lambda_{2}=\bar{\lambda}_{1}$ to include the LHP zeroes. The remaining closed loop poles may be chosen arbitrarily; we will choose $\lambda_{3}=-2.8$ and $\lambda_{4}=-0.7$, since control effort is generally minimized by reducing the movement of stable open loop poles.

Applying the Moore (1976) algorithm, we obtain corresponding closed loop eigenvectors

$v_{1}=\left[\begin{array}{llll}0.147 & 0.065-0.070 i & 0.030-0.100 i & -0.053+\end{array}\right.$ $0.080 i]^{T}, v_{2}=\bar{v}_{1}, v_{3}=\left[\begin{array}{llll}-0.007 & 0.303 & 0.150 & 0.082\end{array}\right]^{T}$ and $v_{4}=[-0.307,-0.321,0.101,0.181]^{T}$, which are chosen to satisfy $C v_{1}=C v_{2}=\left[\begin{array}{ll}0 & 0\end{array}\right]^{T}, C v_{3}=\left[\begin{array}{ll}1 & 0\end{array}\right]^{T}$ and $C v_{4}=\left[\begin{array}{ll}0 & 1\end{array}\right]^{T}$. The feedback gain matrix $F$ is

$$
F=\left[\begin{array}{cccc}
-2.557 & 4.086 & -0.757 & -0.514 \\
2.186 & 1.171 & -2.814 & 8.971
\end{array}\right] \text {. }
$$

The system responses are shown in Figure 1 for several values of the initial condition $x_{0}$. In each case we see that both components of the output trajectory converge to their target value without overshoot.

If we wish to increase the convergence speed, then we may instead choose larger negative eigenvalues $\lambda_{3}=-4$, $\lambda_{4}=-5$. In this case $F$ is

$$
F=\left[\begin{array}{cccc}
-0.7143 & -0.2857 & 4.4286 & 2.1429 \\
1.5714 & 3.4286 & -4.1429 & 9.2857
\end{array}\right] \text {. }
$$




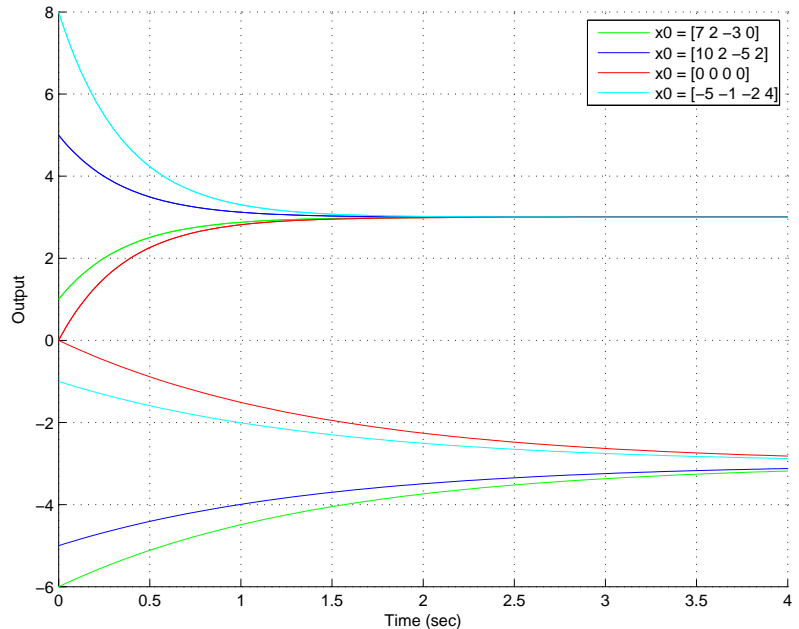

Fig. 1. Minimum phase MIMO system $\Sigma_{1}$.

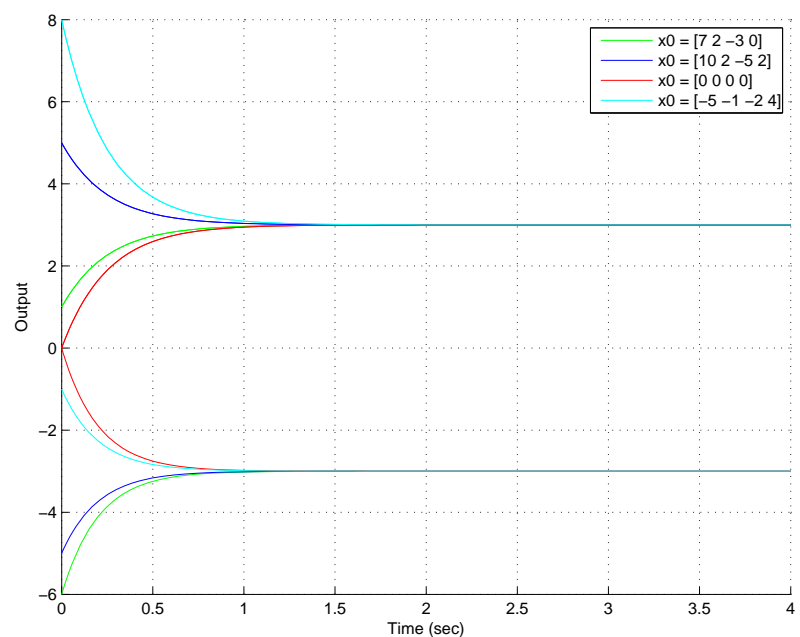

Fig. 2. Minimum phase MIMO system $\Sigma_{1}$.

Example 2: Consider the MIMO system $(m=3, p=2)$ $\Sigma_{2}$ with

$A=\left[\begin{array}{cccc}0 & 6 & 0 & -4 \\ 0 & 1 & 0 & 0 \\ 6 & 0 & 0 & 2 \\ 0 & 10 & 3 & -7\end{array}\right], B=\left[\begin{array}{ccc}4 & 7 & 0 \\ -3 & 0 & 0 \\ 3 & 0 & -10 \\ 0 & 0 & 0\end{array}\right], C^{T}=\left[\begin{array}{cc}0 & -5 \\ -3 & 0 \\ 0 & 9 \\ 6 & -7\end{array}\right]$

Assume that the tracking target is $r=\left[\begin{array}{ll}1 & -2\end{array}\right]^{T}$. We see that the system has no invariant zeroes. The system is right invertible but not left invertible. Also $\mathcal{V}^{\star}=\operatorname{span}\left\{\left[\begin{array}{llll}454 & -70 & 225 & -35\end{array}\right]^{T},\left[\begin{array}{llll}0 & 18 & 7 & 9\end{array}\right]^{T}\right\}$ has dimension $2=n-p$, so we may apply the squaring down algorithm in Lemma 4 to $\Sigma_{2}$. Choosing the assignable internal eigenvalues of $\mathcal{V}^{\star}$ at $z_{1}=-1, z_{2}=-2$ gives

$$
\begin{aligned}
\Phi & =\left[\begin{array}{cccc}
-0.1728 & -5.7527 & -1.6890 & 3.2635 \\
-0.3403 & -7.2990 & -2.9589 & -3.0499 \\
0.5935 & 1.2996 & 1.0118 & 2.1916
\end{array}\right], \\
\Omega & =\left[\begin{array}{cc}
-1 & 0 \\
0 & 7 \\
0 & 18
\end{array}\right]
\end{aligned}
$$

with squared down system $\hat{\Sigma}$ with

$$
\begin{aligned}
\hat{A} & =\left[\begin{array}{cccc}
-3.0734 & -68.1037 & -27.4678 & -12.2954 \\
0.5184 & 18.2581 & 5.0669 & -9.7904 \\
-0.4530 & -30.2541 & -15.1846 & -10.1259 \\
0 & 10.0000 & 3.0000 & -7.0000
\end{array}\right], \\
\hat{B} & =\left[\begin{array}{cc}
-4.0000 & 2.5371 \\
3.0000 & 0 \\
-3.0000 & -9.3200 \\
0 & 0
\end{array}\right], \hat{C}=C,
\end{aligned}
$$

which is left and right invertible with zeros at $z_{1}=-1$, $z_{2}=-2$, as chosen. Hence $\hat{\Sigma}$ has $n-p=2$ LHP zeros and we may apply the algorithm in Theorem 3 . The closed loop poles are chosen to include the zeros $\lambda_{1}=-1, \lambda_{2}=-2$, and we arbitrarily choose $\lambda_{3}=-3, \lambda_{4}=-4$. We obtain the feedback gain matrix

$$
\hat{F}=\left[\begin{array}{cccc}
0.1728 & 0.4194 & -0.3110 & -0.5968 \\
0.0777 & -0.0121 & -0.1398 & 0.1329
\end{array}\right] .
$$

Several output trajectories are shown in Figure 3.
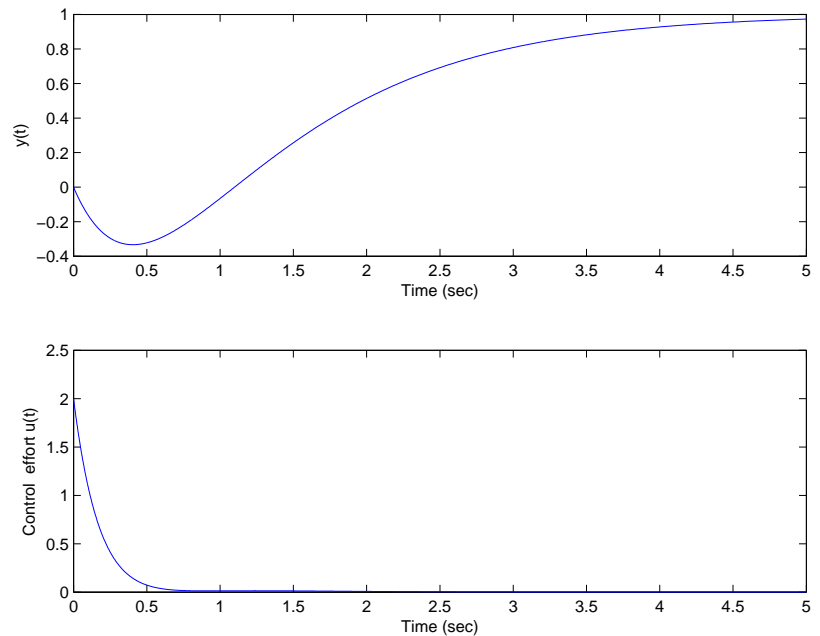

Fig. 3. Non square minimum phase system $\Sigma_{2}$.

Example 3: Here we consider the SISO $(m=p=1)$ nonminimum phase system $\Sigma_{3}$ with

$A=\left[\begin{array}{cccc}0 & 1 & 0 & 0 \\ 0 & 0 & 1 & 0 \\ 0 & 0 & 0 & 1 \\ 0 & -5 & -9 & -5\end{array}\right], B=\left[\begin{array}{l}0 \\ 0 \\ 0 \\ 1\end{array}\right], C=\left[\begin{array}{llll}15 & -7 & -7 & 1\end{array}\right]$,

which was discussed in Bement and Jayasuriya (2004). The authors used an eigenvalue assignment method to obtain a state feedback controller that would track a target reference of $r=1$ from an initial condition of $x_{0}=\left[\begin{array}{llll}0 & 0 & 0 & 0\end{array}\right]^{T}$ without overshoot.

The poles and zeros of $\Sigma_{3}$ are $\{0,-1,-2 \pm i\}$ and $\{1,-3,-5\}$ respectively. Hence $\Sigma_{2}$ has $n-2 p=2$ LHP zeros, and we may use the Theorem 3 to design a nonovershooting controller. We choose closed loop eigenvalues $\lambda_{1}=-5, \lambda_{2}=-3, \lambda_{3}=-2, \lambda_{4}=-1$. Applying the algorithm in Moore (1976) gives a state feedback gain matrix of $F=\left[\begin{array}{llll}30 & 56 & 32 & 6\end{array}\right]$ with closed loop eigenvectors

$v_{1}=\left[\begin{array}{llll}0.0078 & 0.0349 & 0.1111 & 0.0625\end{array}\right]^{T}$,

$v_{2}=\left[\begin{array}{llll}-0.0392 & -0.1048 & -0.2222 & -0.0625\end{array}\right]^{T}$,

$v_{3}=v_{1,1}=\left[\begin{array}{llll}0.1960 & 0.3143 & 0.4444 & 0.0625\end{array}\right]^{T}$, 
$v_{4}=v_{1,2}=\left[\begin{array}{llll}-0.9798 & -0.9429 & -0.8889 & -0.0625\end{array}\right]^{T}$, and hence

$$
\mathcal{L}_{1}=\left\{\gamma_{1,1} v_{1,1}+\gamma_{1,2}\left(v_{1,1}-v_{1,2}\right): \gamma_{1,1} \gamma_{1,2} \leq 0\right\}
$$

Solving (4) for $\Sigma_{3}$ gives $x_{s s}=\left[\begin{array}{llll}1 / 15 & 0 & 0 & 0\end{array}\right]^{T}$. To see if $\Sigma_{c l}$ is non overshooting for $x_{0}=0$, we apply the test in Remark 3.3 and obtain

$$
\begin{aligned}
\gamma & =\left[v_{1}\left|v_{2}\right| v_{1,1} \mid v_{1,1}-v_{1,2}\right]^{-1}\left(x_{s s}-x_{0}\right) \\
& =\left[\begin{array}{cccc}
-0.0078 & 0.0349 & 0.1111 & 0.0486 \\
0.0392 & -0.1048 & -0.2222 & -0.1597 \\
-0.1960 & 0.3143 & 0.4444 & 0.3819 \\
0.9798 & -0.9429 & -0.8889 & -0.8264
\end{array}\right]\left[\begin{array}{c}
1 / 15 \\
0 \\
0 \\
0
\end{array}\right] \\
& =\left[\begin{array}{llll}
-0.0005 & 0.0026 & -0.0131 & 0.0653
\end{array}\right]^{T} .
\end{aligned}
$$

Since the product of the last two coefficients is negative, we see that the system response will be non overshooting for $x_{0}=0$. The output $y(t)$ is plotted in Figure 4 . Note that the trajectory exhibits undershoot, as expected for systems with a zero on the positive real axis Darbha (2003).

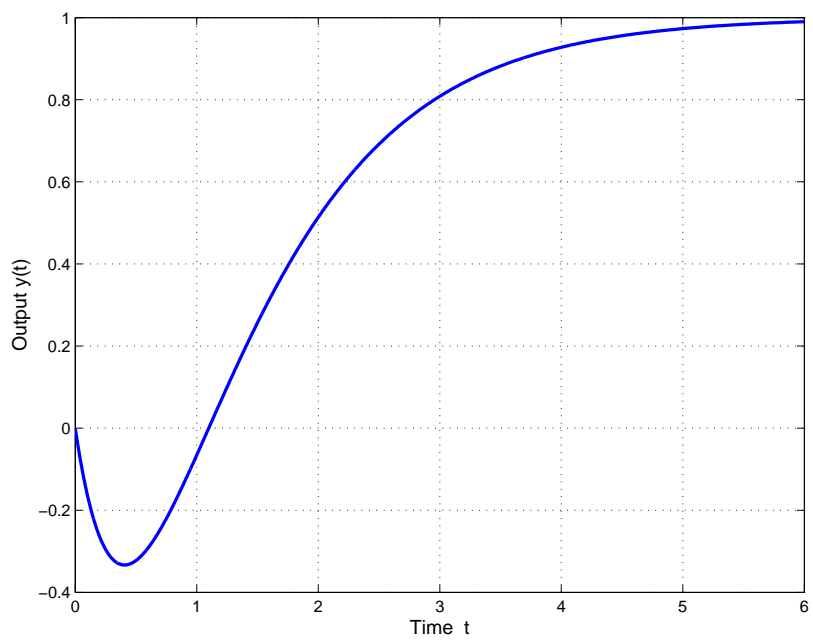

Fig. 4. Nonminimum phase SISO system $\Sigma_{3}$.

Some key features of the above method, in comparison with Bement and Jayasuriya (2004) are

(1) Both methods apply to systems $\Sigma$ with $n-2$ LHP zeroes. However, for Bement and Jayasuriya (2004) the LHP zeroes must be real; in our method these are permitted to be complex.

(2) The Bement and Jayasuriya (2004) algorithm yields a non-overshooting controller from zero initial conditions. The above algorithm identifies a set of initial conditions from which the controller will give a nonovershooting response. To ensure that $x_{0}=0$, or any other desired initial condition, will yield a nonovershooting response, we must select the eigenvalues $\lambda_{3}$ and $\lambda_{4}$ such that $x_{0}$ satisfies the test in Remark 3.3 .

(3) The Bement and Jayasuriya (2004) algorithm requires careful hand tuning from the controller designer to choose appropriate eigenvalues. Conversely, the Moore (1976) algorithm can readily be executed in MATLAB and the only design decision is the selection of the eigenvalues $\lambda_{3}$ and $\lambda_{4}$. These may be tuned to achieve faster/slower convergence with correspond- ing stronger/weaker control effort, as required to meet any desired convergence rate or actuator constraints.

\section{CONCLUSION}

A design method for an LTI state feedback tracking controller to achieve a nonovershooting step response has been introduced. To the best of the author's knowledge, this is the first such control scheme to consider MIMO systems, and non-zero initial conditions.

\section{ACKNOWLEDGEMENTS}

The authors would like to thank Ben Chen from the National University of Singapore for bringing the paper Moore (1976) to their attention.

\section{APPENDIX}

Lemma 6. Let $\lambda_{1}<\lambda_{2}<0$ and let

$$
y(t)=\alpha_{1} e^{\lambda_{1} t}+\alpha_{2} e^{\lambda_{2} t}
$$

Then $y(t)$ will not change sign for any $t \geq 0$ if and only if

$$
\left(\alpha_{1}, \alpha_{2}\right)=\left(\gamma_{1}+\gamma_{2},-\gamma_{2}\right)
$$

for some real numbers $\gamma_{1}, \gamma_{2}$ with $\gamma_{1} \gamma_{2} \leq 0$.

Lemma \%. If the system $\Sigma$ is right-invertible, every uncontrollable eigenvalue of $\Sigma$ is also an invariant zero of $\Sigma$.

\section{REFERENCES}

G. Basile and G. Marro. Controlled and Conditioned Invariants in Linear System Theory. Prentice Hall, Englewood Cliffs, New Jersey, 1992.

Bement, M. and S. Jayasuriya, Use of state feedback to achieve a nonovershooting step response for a class of nonminimum phase systems, Journal of Dynamical Systems, Measurement and Control 126 pp. 657-660.

Darbha, S., On the synthesis of controllers for continuous time LTI systems that achieve a non-negative impulse response, Automatica 39 (2003) pp. 159-165.

Darbha, S. and S.P. Bhattacharyya, On the synthesis of controllers for a nonovershooting step response, IEEE Transactions on Automatic Control 48 (2003) pp. 797799.

Darbha, S. and S.P. Bhattacharyya, Controller synthesis for sign invariant impulse response, IEEE Transactions on Automatic Control 47 (2002) pp. 1346-1351.

Krstic, M. and Bement, M., Nonovershooting control of strict feedback nonlinear systems, IEEE Transactions on Automatic Control 51 (2006) pp. 1938-1943.

Moore, B.C., On the Flexibility Offered by State Feedback in Multivariable systems Beyond Closed Loop Eigenvalue Assignment, IEEE Transactions on Automatic Control (1976) pp. 689-692.

Maciejowski, J., Multivariable Feedback Design, (1989) Addison-Wesley.

L. Ntogramatzidis, and D. Prattichizzo. Squaring Down LTI Systems: A Geometric Approach. Systems \& Control Letters, 56(3):236-244, 2007. 